\title{
Effect of amiloride and saline on nasal \\ mucociliary clearance and potential difference in cystic fibrosis and normal subjects
}

\author{
P G Middleton, D M Geddes, E W F W Alton
}

\begin{abstract}
Background-Mucociliary clearance is an important component of pulmonary defence. Maximum clearance is thought to depend on an optimal depth of the sol layer, allowing the most efficient interaction between the cilia and the overlying mucus layer. Sodium absorption, the major ion transport in human airways, is thought to be important in the regulation of the depth of the sol layer. In the airways of patients with cystic fibrosis sodium absorption is increased and mucociliary clearance decreased. Amiloride, a sodium channel blocker, has been shown to improve pulmonary mucociliary clearance in patients with cystic fibrosis. However, its effects on nasal mucociliary clearance in either normal subjects or those with cystic fibrosis are unknown. A study was therefore performed to investigate whether nebulised amiloride improves nasal mucociliary clearance in normal or cystic fibrosis subjects.
\end{abstract}

Methods-Nasal mucociliary clearance was measured by the saccharin clearance technique in 12 normal subjects and 12 with cystic fibrosis. For the control study measurements were made on two consecutive days and the mean time for each subject averaged. For the drug study measurements were also made on two consecutive days, after administration of nasally nebulised amiloride or placebo (saline) in a double blind manner. Nasal potential difference was measured in eight patients with cystic fibrosis after the administration of amiloride or placebo to assess the efficacy of deposition and duration of action.

Results-Baseline values of mucociliary clearance were significantly faster in the normal subjects than in those with cystic fibrosis. In both groups mucociliary clearance was increased after both saline and amiloride, with no significant difference between either treatment. As previously reported, baseline nasal potential difference was significantly more negative in the subjects with cystic fibrosis. Amiloride significantly reduced the potential difference for at least $60 \mathrm{~min}$ utes in these subjects.

Conclusions-Nebulised saline signifi- cantly improves nasal mucociliary clearance in both normal subjects and those with cystic fibrosis. Amiloride did not appear to exert any additional effects in either group of subjects, despite evidence of its efficacy of deposition.

(Thorax 1993;48:812-816)

Mucociliary clearance is an important component of the pulmonary defence system. Normal function is thought to depend on the amount and viscoelastic properties of the airway surface liquid, together with the number and function of the cilia. A defect in mucociliary transport has long been implicated in the pathogenesis of lung disease in cystic fibrosis. ${ }^{1}$ More recently, the genetic defect in cystic fibrosis has been linked to altered regulation of epithelial chloride channels in response to the cAMP-mediated second messenger pathway, ${ }^{2}$ whilst in airway epithelium there is also increased reabsorption of sodium. ${ }^{3}$ It is hypothesised that these changes lead to the reduction in water content of airway secretions found in cystic fibrosis. ${ }^{4}$ Relative airway dehydration may affect either component of the airway surface liquid, the mucus (gel) layer or the periciliary fluid (sol) layer. Dehydration of the gel layer may result in impaired viscoelastic properties of the mucus, while drying of the sol layer may disrupt the interactions between cilia and mucus. ${ }^{5}$ Either or both of these effects could impair mucociliary clearance, leading to chronic infection and bronchiectasis.

The transepithelial potential difference is increased (more negative) in the airways of patients with cystic fibrosis, principally because of the increased sodium absorption. ${ }^{6}$ Amiloride, a drug which blocks sodium channels, ${ }^{7}$ therefore produces a significantly greater reduction in airway potential difference in patients with cystic fibrosis than in normal subjects. Initial studies of amiloride showed that, after oral administration, insufficient levels were found in the airways to affect ion transport. ${ }^{8}$ Direct delivery to the airways via nebulisation in sheep achieved adequate levels to alter potential difference. ${ }^{9}$ Studies in subjects with cystic fibrosis have shown that nebulised amiloride improves pulmonary mucociliary clearance, both acutely ${ }^{10}$ and over a three week trial period. ${ }^{11}$ However, 
measurements of pulmonary mucociliary clearance in adults with cystic fibrosis are complicated by the presence of bronchiectasis and retained secretions in the airways. In contrast, the respiratory epithelium of the nose demonstrates the cystic fibrosis bioelectric defect, ${ }^{3}$ but is less affected by anatomical abnormalities and retained secretions. Studies of nasal mucociliary clearance may therefore provide a more direct measure of the link(s) between mucociliary clearance and ion transport defects in patients with cystic fibrosis.

The saccharin clearance test, first proposed by Andersen et al, ${ }^{12}$ has been widely used to investigate nasal mucociliary clearance. This method was used to study whether amiloride improves mucociliary clearance more than diluent (saline) in patients with cystic fibrosis or normal subjects. The duration of the effect of amiloride was confirmed by measurement of nasal potential difference.

\section{Methods}

SUBJECTS

Twelve non-smoking subjects of mean age 27 (range 21-42) years with no history of nasal surgery or trauma, asthma, allergic rhinitis, or sinusitis were studied as normal controls. They were taking no medication other than the oral contraceptive. Subjects with cystic fibrosis with no history of nasal surgery or trauma or chronic sinusitis were recruited. All had abnormal sweat tests and clinical features suggestive of cystic fibrosis. In the baseline study 12 patients with cystic fibrosis of mean age 21 (range 16-30) years, forced expiratory volume in one second $\left(\mathrm{FEV}_{1}\right) 39 \%$ predicted (range 17-85\%), and forced vital capacity (FVC) $59 \%$ predicted (range 30-101\%) were studied. For practical reasons the drug study was performed on a second group of 12 subjects with cystic fibrosis having very similar characteristics: (mean age 25 (range 19-36) years, mean $\mathrm{FEV}_{1} 36 \%$ predicted (range 12-82), and mean FVC $56 \%$ predicted (range 21-114)). Normal medications for the baseline and drug study groups were very similar and included inhaled $\beta_{2}$ sympathomimetics (baseline 12, drug study 12), systemic $\beta_{2}$ sympathomimetics (baseline 4 , drug study 4 ), theophylline (baseline 2 , drug study 3 ), oral prednisolone (baseline 2, drug study 2), inhaled corticosteroids (baseline 8, drug study 8 ), inhaled antibiotics (baseline 6 , drug study 8), and pancreatic supplements (baseline 11 , drug study 11). No subjects were taking intranasal corticosteroids. No medications were changed within the 48 hours before study. Sputum culture showed chronic colonisation with Staphylococcus aureus (four patients from each group), Pseudomonas aeruginosa (11 patients from each group), and Pseudomonas cepacia (one patient from each group). All subjects gave informed consent and the trial was approved by the Ethics Committee of the hospital.

MEASUREMENT OF MUCOCILIARY CLEARANCE Nasal mucociliary clearance was measured by the saccharin clearance test described by
Andersen et al. ${ }^{12}$ Testing was performed at least four weeks after the subjects had last suffered from an upper respiratory tract infection. For the normal and both groups of subjects with cystic fibrosis, baseline and drug tests were performed on two successive days. Before the first test the patency of both nostrils was assessed by direct vision, with the more patent nostril being used for the entire study. Subjects were instructed to refrain from taking substances containing caffeine for at least four hours before each test, and in the subjects with cystic fibrosis the test was performed at least four hours after the last inhaled medication. Before each test the subjects spent at least one hour in a constant environment at a temperature of $20-24^{\circ} \mathrm{C}$ and a relative humidity of $30-50 \%$, with all tests for each subject performed in the same room. The nasal mucosa was inspected before each test, and a $1 \mathrm{~mm}$ particle of a commercially available saccharin tablet (Sweetex) was then placed under direct vision on the lateral aspect of the floor of the nose, approximately $5 \mathrm{~mm}$ behind the anterior end of the inferior turbinate. The subjects remained seated, breathing normally through their nose and mouth with their head inclined approximately $10^{\circ}$ forward. They were instructed to swallow every 30 seconds, but not to blow their nose, sniff, sneeze, eat or drink during the test. The time to first tasting of the saccharin was recorded in seconds.

In the drug study, tests were performed immediately after administration of nasally nebulised saline or amiloride in a double blind protocol. Nebulisation for five minutes using nasal prongs attached to an ultrasonic nebuliser (Fisoneb) delivered a total volume of 4-4.5 ml. After nebulisation the subjects gently blew their nose to remove any excess secretions and the saccharin test was commenced immediately.

\section{MEASUREMENT OF NASAL POTENTIAL} DIFFERENCE

In eight subjects with cystic fibrosis from the drug group the effect of amiloride on nasal potential difference was measured using a previously described method. ${ }^{13}$ Briefly, the exploring electrode consisted of a modified 8 Fr Foley catheter filled with an equal mixture of Ringer's lactate and ECG electrode cream connected to a high impedance voltmeter by a silver/silver chloride electrode. The reference electrode consisted of a second silver/ silver chloride electrode placed over an area of abraded skin on the forearm, again connected to the voltmeter. Before the recordings the offset of the electrodes was measured and appropriate corrections made to recorded values.

The catheter, with the balloon deflated, was slowly passed along the floor of the nose and the maximum nasal potential difference in each nostril was recorded and averaged to give a single value for a given time point. (For discussion purposes increases and decreases refer to the absolute magnitude of potential difference.) 
After three control readings (on each side) nasally nebulised saline or amiloride ( $1 \mathrm{mmol} / \mathrm{l}$ ) was administered, again using a double blind protocol. Readings were repeated at $5,15,30,45$, and 60 minutes after each nebulisation.

\section{STATISTICAL ANALYSIS}

Comparison of the saccharin clearance time within the normal subjects was made using the Wilcoxon signed rank test. As the subjects with cystic fibrosis were different in the baseline and drug studies, comparisons were made using the Mann-Whitney $U$ test. Comparison of the nasal potential difference before and after nebulised drugs was performed using the Wilcoxon signed rank test and between treatments using the MannWhitney $U$ test. The null hypothesis was rejected at $\mathrm{p}<0.05$. Data are presented as mean (SE) for convenience.

\section{Results}

SACCHARIN CLEARANCE TIME

In the normal subjects $(n=12)$ the mean (SE) results of replicate tests of saccharin clearance time were 568 (40) seconds and 627 (46) seconds ( $p=$ NS) on two consecutive days. Both saline and amiloride significantly reduced the saccharin clearance time to 466 (51) seconds and 458 (44) seconds respectively $(p<0.05)$; the difference between the two drugs was not significant $(p=$ 0.95 ) (fig 1).

In the subjects with cystic fibrosis $(n=12)$ the mean (SE) results of replicate tests of baseline saccharin clearance time was 1640 (402) and 1554 (222) seconds ( $p=N S$ ). Saline significantly decreased the saccharin clearance time to 959 (157) seconds, ( $p<$ 0.05 ), whilst amiloride decreased it significantly to $786(210)$ seconds $(p<0.01)$. Comparison of the saccharin clearance time after the two drugs was not significantly different $(p=0 \cdot 19)$ (fig 1).

Comparison of the baseline tests between the two groups of subjects showed that saccharin clearance time in the subjects with cystic fibrosis was significantly slower than in the normal subjects $(p<0.0001)$.

Figure 1 Saccharin clearance time in the normal subjects: $(\square)$ baseline, $(O)$ after saline, and $(\triangle)$ after amiloride, and in the subjects with cystic fibrosis: (1) baseline, (†) after saline, (৯) after amiloride. $\star_{p}<0.05, \star_{p}<0.01$ compared with baseline for that group; \# $p<0.0001$ compared with baseline in the normal subjects.

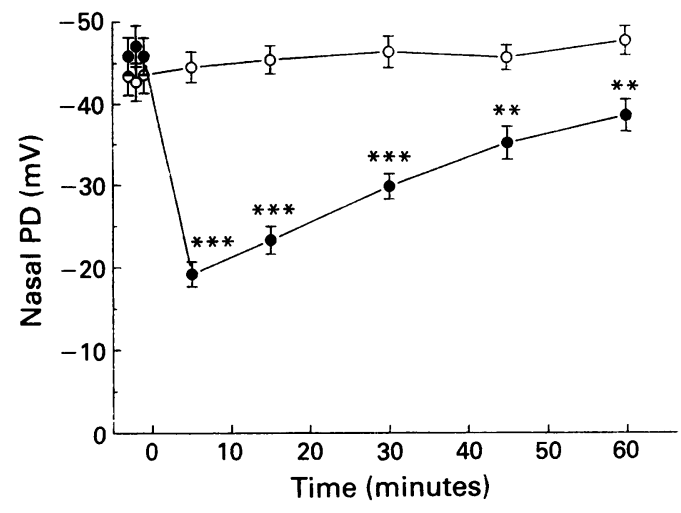

Figure 2 Nasal potential difference measured in the subjects with cystic fibrosis following either saline $(O)$ or amiloride $(\mathbf{O})$ given at time $0 .{ }^{* \star *} p<0.001$, $\star_{p}<0.01$ compared with saline.

\section{NASAL POTENTIAL DIFFERENCE}

The mean (SE) baseline nasal potential dif- $\infty$ ference in the eight subjects with cystic fibrosis was $-43.2(1 \cdot 3) \mathrm{mV}$ and $-46 \cdot 3(1 \cdot 3) \mathrm{mV}$ on the saline and amiloride days respectively $(p=N S)$. Nasally nebulised saline caused no significant change in the nasal potential difference at 5,15 , or 45 minutes, but caused a small, significant $(p<0.05)$ increase of -3.1 $\mathrm{mV}$ at 30 minutes and $-4.4 \mathrm{mV}$ at $60 \mathrm{~min}$ utes. Amiloride consistently diminished the nasal potential difference in all eight cystic fibrosis subjects to a mean potential difference of $-19.2(1.5) \mathrm{mV}$ at five minutes $(\mathrm{p}<$ 0.001 compared with saline). The potential difference then slowly increased (more negative) to a value of $-38.5(2.0) \mathrm{mV}$ at $60 \mathrm{~min}-$ utes, which was still significantly reduced from the saline values ( $p<0.01$ ) (fig 2).

\section{Discussion}

For measurement of saccharin clearance time we slightly modified the method of Andersen et $a l^{12}$ by placing the saccharin particle on the floor of the nose, allowing the same area of nasal mucosa to be used for both the saccharin clearance and nasal potential difference measurements. Previous work has shown that measurement of mucociliary clearance on the floor of the nose is as reproducible as that on the inferior turbinate. ${ }^{14}$ This was confirmed by the current baseline measurements of saccharin clearance time in the normal subjects and those with cystic fibrosis. As expected, saccharin clearance time was significantly longer in the subjects with cystic fibrosis, with wider variability both within and between subjects, confirming previous findings. ${ }^{15}$ The mean baseline values were similar to those in previous reports (normal: 702 seconds, ${ }^{16} 840$ seconds, ${ }^{17}$ and 606 seconds ${ }^{15}$; cystic fibrosis: 1896 seconds $^{15}$ ).

In both normal subjects and those with cystic fibrosis saline and amiloride increased nasal mucociliary clearance (shorter saccharin clearance time). Neither group showed a difference between the two agents. The apparent lack of effect of amiloride was not due to a delivery problem as the nasal potential dif- 
ference was decreased by $59 \%$ in the subjects with cystic fibrosis five minutes after the end of nebulisation and continued to decrease the nasal potential difference for at least $60 \mathrm{~min}$ utes. Previous studies of the effect of amiloride on respiratory epithelia in vivo are limited. In sheep nebulised amiloride significantly reduced the potential difference for less than 30 minutes $^{9}$ with a half life of approximately 10.5 minutes. More recently, in the human trachea, nebulised amiloride has been shown to have a half life of approximately 40 minutes, although measurements of the potential difference were not reported. ${ }^{18} \mathrm{We}$ are unaware of any other studies of the time course of the effect of amiloride on the potential difference in the human respiratory tract in vivo.

As there was a small but insignificant trend to faster mucociliary clearance in the subjects with cystic fibrosis after amiloride, we considered whether the other medications taken by these patients may have been important. All tests were performed at least four hours after inhaled bronchodilators or antibiotics, but five of the subjects were also taking at least one form of systemic bronchodilator. Subgroup analysis showed that amiloride improved mucociliary clearance by at least 30 seconds in three of these five subjects and in three of the other seven subjects.

It would appear, therefore, that amiloride ( $1 \mathrm{mmol} / \mathrm{l}$ in saline) offers no benefit over saline in improving mucociliary clearance in the nose. Although amiloride has been shown to improve pulmonary mucociliary clearance in subjects with cystic fibrosis, there have been no previous reports of the effect of amiloride on nasal mucociliary clearance. Köhler et al showed that nebulised amiloride increased clearance of radioactively labelled sputum in 10 of 14 subjects with cystic fibrosis 30 minutes after inhalation of amiloride. ${ }^{10}$ In an extension of this study App et al reported that the main effect of amiloride occurred within the first 10 minutes. ${ }^{11}$ Knowles et $a l^{19}$ have also shown that, in subjects who are taken off all other therapy, amiloride lessens the rate of decline in lung function over a 25 week period. Sputum collected during amiloride treatment showed improved indices of clearance.

More recently, however, Graham et al have shown that the addition of amiloride to existing treatment resulted in no significant changes in either lung function or sputum rheology over a similar time period. Thus, although amiloride improves pulmonary mucociliary clearance in subjects taken off all other treatment, it may exert less effect on subjects who continue on their normal medications.

There are a number of possible mechanisms that could account for the present finding of increased mucociliary clearance following nasally nebulised saline. Firstly, the depth of the sol layer may have been increased. Although it is generally believed that the depth of the sol layer is optimal in the bronchial tree, in the nose it may be decreased as a result of drying by inspired air; nebulised saline may increase the depth of the sol layer, allowing better interactions between mucus and cilia. The gel layer may also have been affected: gentle blowing of the nose following nebulisation may have reduced its volume and any remaining mucus would have been hydrated, improving its viscoelastic properties and thus its clearance.

Ciliary beat frequency may also have increased mucociliary clearance as nebulised saline has been shown to induce a small but significant increase in ciliary beat frequency in vivo, ${ }^{21}$ although this effect has not been found in vitro. ${ }^{22}$ We suggest that the most likely reason for the increased mucociliary clearance after saline is an increase in the depth of the sol layer allowing better interaction between cilia and mucus. However, as it is generally accepted that the applied saccharin moves in both the sol and gel layers, ${ }^{23}$ we cannot discount the possibility of an effect on the gel layer.

It is unlikely that the increased mucociliary clearance after saline was simply the result of saccharin movement through the nose on gravity dependent fluid flow, as the volume of nebulised fluid remaining in the nose after nebulisation was minimised and, during the test itself, the subject remained seated with the head inclined $10^{\circ}$ forward so that the nasal floor was sloping upwards posteriorly. Any gravitational fluid flow would therefore have been anterior, against the active transport.

In conclusion, inhaled saline and amiloride significantly improved nasal mucociliary clearance as measured by the rate of saccharin clearance. Although ultrasonically nebulised amiloride significantly reduced the nasal potential difference for a period of at least 60 minutes in subjects with cystic fibrosis, it had no significant additional effect on mucociliary clearance in either the normal subjects or those with cystic fibrosis.

We would like to thank the subjects who took part in this study. PGM is a Cystic Fibrosis Research Trust Fellow and EWFWA a British Heart Foundation Intermediate Fellow. The study was supported by the British Medical Association HC Roscoe Fellowship, the Cystic Fibrosis Research Trust, and the British Lung Foundation.

1 Yeates DB, Sturgess JM, Kahn SR, Levison H, Aspin N. Mucociliary transport in trachea of patients with cystic fibrosis. Arch Dis Child 1976;51:28-33.

2 Egan M, Flotte T, Afione S, Solow R, Zeitlin PL, Carter $\mathrm{BJ}$, et al. Defective regulation of outwardly rectifying $\mathrm{Cl}^{-}$ channels by protein kinase A corrected by insertion of CFTR. Nature 1992;358:581-4.

3 Knowles M, Gatzy J, Boucher R. Increased bioelectric potential difference across respiratory epithelia in cystic fibrosis. N Engl ₹ Med 1981;305:1489-95.

4 Potter JL, Matthews LW, Spector S, Lemm J. Studies on pulmonary secretions. II. Osmolality and the ionic environment of pulmonary secretions from patients with cystic fibrosis, bronchiectasis, and laryngectomy. $\mathrm{Am}$ Rev Respir Dis 1967;96:83-7.

5 Quinton PM. Cystic fibrosis: a disease in electrolyte transport. FASEB ₹ 1990;4:2709-17.

6 Willumsen NJ, Boucher RC. Sodium transport and intracellular sodium activity in cultured human nasal epithelium. Am F Physiol Cell Physiol 1991;261:C319-31.

7 Kleyman TR, Cragoe EJ Jr. Amiloride and its analogs as tools in the study of ion transport. $7 \mathrm{Membr}$ Biol 1988;105:1-21. 
8 Knowles MR, Church NL, Waltner WE, Yankaskas JR, Gilligan $\mathrm{P}, \mathrm{King} \mathrm{M}$, et al. Aerosolized amiloride as treatment of cystic fibrosis lung disease: a pilot study. $A d v$ Exp Med Biol 1991;290:119-32.

9 Mentz WM, Brown JB, Friedman M, Stutts MJ, Gatzy JT, Boucher RC. Deposition, clearance, and effects of aerosolized amiloride in sheep airways. Am Rev Respir Dis 1986;134:938-43.

10 Köhler D, App E, Schmitz-Schumann M, Würtemberger $G$, Matthys $H$. Inhalation of amiloride improves the mucociliary and the cough clearance in patients with cystic fibroses. Eur $\mathcal{f}$ Respir Dis 1986;69(Suppl 146): cystic fibro

11 App EM, King M, Helfesrieder R, Köhler D, Matthys H Acute and long-term amiloride inhalation in cystic fibrosis lung disease. A rational approach to cystic fibrosis therapy. Am Rev Respir Dis 1990;141:605-12

12 Andersen I, Camner P, Jensen PL, Philipson K, Proctor DF. A comparison of nasal and tracheobronchial clearance. Arch Environ Health 1974;29:290-3.

13 Alton EWFW, Currie D, Logan-Sinclair R, Warner JO, Hodson ME, Geddes DM. Nasal potential difference: a clinical diagnostic test for cystic fibrosis. Eur Respir $\mathfrak{f}$ 1990;3:922-6.

14 Quinlan MF, Salman SD, Swift DL, Wagner HN Jr, Proctor DF. Measurement of mucociliary function in man. Am Rev Respir Dis 1969;99:13-23.

15 Rutland J, Cole PJ. Nasal mucociliary clearance and ciliary beat frequency in cystic fibrosis compared with sinusitis and bronchiectasis. Thorax 1981;36:654-8.
16 Stanley PJ, Wilson R, Greenstone MA, Mackay IS, Cole PJ. Abnormal nasal mucociliary clearance in patients with rhinitis and its relationship to concomitant chest disease. Br $\mathcal{F}$ Dis Chest 1985;79:77-82.

17 Stanley P, MacWilliam L, Greenstone M, Mackay I, Cole P. Efficacy of a saccharin test for screening to detect abnormal mucociliary clearance. Br $\mathcal{F}$ Dis Chest 1984; 78:62-5

18 Waltner WE, Church NL, Gatzy J, Boucher R, Knowles $M$. Deposition, pharmacokinetics, and toxicity of amiloride in normal and cystic fibrosis (CF) subjects. Am Rev Respir Dis 1987;135:A288.

19 Knowles MR, Church NL, Waltner WE, Yankaskas JR, Gilligan P, King $M$, et al. A pilot study of aerosolized amiloride for the treatment of lung disease in cystic fibrosis. N Engl f Med 1990;322:1 189-94.

20 Graham A, Hasani A, Alton EWFW, Martin GP, Marriott C, Hodson ME, et al. A clinical trial of nebulised amiloride in patients with cystic fibrosis. Eur Respir $\mathcal{F}$ 1993 (in press).

21 Wong LB, Miller IF, Yeates DB. Pathways of substance P stimulation of canine tracheal ciliary beat frequency. f Appl Physiol 1991;70:267-73.

22 Rutland J, Griffin W, Cole P. Nasal brushing and measurement of ciliary beat frequency. An in vitro method for evaluating pharmacological effects on human cilia. Chest 1981;80(Suppl):865-7.

23 Puchelle E, Aug F, Pham QT, Bertrand A. Comparison of three methods for measuring nasal mucociliary clearance in man. Acta Otolaryngol 1981;91:297-303. 